\title{
Matthieu BÉRA \& Nicolas SEMBEL (dir.), Les Formes élémentaires de la vie religieuse, cent ans après. Émile Durkheim et la religion
}

Matthias Fringant

\section{(2) OpenEdition Journals}

Édition électronique

URL : https://journals.openedition.org/ress/7725

DOI : $10.4000 /$ ress. 7725

ISBN : 1663-4446

ISSN : $1663-4446$

Éditeur

Librairie Droz

Édition imprimée

Date de publication : 6 décembre 2021

Pagination : 336-339

ISSN : 0048-8046

Référence électronique

Matthias Fringant, « Matthieu BÉRA \& Nicolas SEMBEL (dir.), Les Formes élémentaires de la vie religieuse, cent ans après. Émile Durkheim et la religion », Revue européenne des sciences sociales [En ligne], 59-2 | 2021, mis en ligne le 01 décembre 2021, consulté le 09 décembre 2021. URL : http:// journals.openedition.org/ress/7725; DOI : https://doi.org/10.4000/ress.7725

Ce document a été généré automatiquement le 9 décembre 2021

(c) Librairie Droz 


\title{
Matthieu BÉRA \& Nicolas SEMBEL (dir.), Les Formes élémentaires de la vie religieuse, cent ans après. Émile Durkheim et la religion
}

\author{
Matthias Fringant
}

\section{RÉFÉRENCE}

Matthieu BÉRA \& Nicolas SEMBEL (dir.), 2019, Les Formes élémentaires de la vie religieuse, cent ans après. Émile Durkheim et la religion, Paris, Classiques Garnier, 456 p.

1 Le centenaire de la publication par Émile Durkheim en 1912 des Formes élémentaires de la vie religieuse (abrévié Formes dans la suite de ce compte rendu) a entrainé un certain nombre de commémorations. Pour s'en tenir à l'espace français, en 2012, deux numéros spéciaux de revues ont paru (L'Année sociologique, 62-2, 2012 ; Archives de sciences sociales des religions, 159, 2012), et un colloque organisé par Matthieu Béra et Nicolas Sembel s'est tenu à Bordeaux, Pessac, et Talence. Le livre dont il est ici question est le recueil des communications effectuées lors de cette manifestation scientifique.

2 L'ouvrage débute par une courte introduction rédigée par Matthieu Béra. L'auteur présente en premier lieu les raisons chronologiques, thématiques, et méthodologiques qui expliquent le «statut à part» (p. 11) des Formes - et qui donc justifient la nécessité de son étude - avant de présenter synthétiquement l'objet, la structure, et le contenu $\mathrm{du}$ livre. Celui-ci se poursuit avec treize contributions réparties en trois parties thématiques distinctes, et s'achève par une conclusion rédigée par Nicolas Sembel. La première partie de l'ouvrage contient cinq textes ayant trait aux premières réceptions des Formes, la seconde quatre lectures susceptibles de fournir des ressources pour la pratique sociologique actuelle, et la troisième quatre recherches sur les sources utilisées au cours de l'élaboration des Formes. Le volume présente également un 
appareil critique rigoureux et utile : une notice concernant les abréviations utilisées, un avertissement explicitant l'édition des Formes utilisée par les auteurs, un index des noms propres, un résumé des différentes contributions, une table des figures, et enfin une table des matières.

Les cinq premiers textes viennent utilement compléter le périmètre géographique, disciplinaire, et thématique déjà traité par les études de réception présentes au sein des deux numéros spéciaux cités. Ceux-ci se centraient alors sur la réception française du livre parmi les spécialistes de religion, les philosophes, et les opposants à Durkheim.

En ce qui concerne l'extension géographique, les chapitres de François Pizzaro Noël et de Didier Lapeyronnie - décédé en 2020 - fournissent des éléments empiriques sur deux séquences distinctes de la réception nord-américaine de l'ouvrage. En se centrant spécifiquement sur deux comptes rendus rédigés par Robert E. Park et Charles E. Gehlke pendant la période 1912-1930, François Pizzaro Noël met en évidence les tendances lourdes au nominalisme et à l'individualisme dans la sociologie étatsunienne. Celles-ci ont préparé la plus célèbre réception de Durkheim par Talcott Parsons en 1937 dans The Structure of Social Action. Didier Lapeyronnie s'intéresse pour sa part à la réception plus tardive du livre dans la "microsociologie contemporaine " (p. 105), notamment à travers les thèmes de la sociologie des petites villes, de l'analyse des rituels d'interaction, et de l'interprétation des relations sexuelles. L'auteur insiste ici sur le rôle qu'a joué le sociologue Erving Goffman dans cette appropriation du traitement durkheimien des rituels pour l'analyse des interactions sociales, approche reprise par la suite par Randall Collins.

Plutôt que de porter l'attention sur l'opposition à Durkheim, Jean-Christophe Marcel signe un court texte sur la "non-réception» (p.32) des Formes par celui qui fut au contraire un des plus proches de l'auteur, son neveu Marcel Mauss. Si trois séquences relativement distinctes de cette réception hésitante sont bien identifiées (une première suivant la parution des Formes, une seconde à partir de 1918, et une troisième débutant en 1923-1924 suite à la parution du célèbre essai sur le don), il nous semble cependant que leur explication aurait pu être davantage approfondie.

6 Concernant la réception dans d'autres disciplines, les textes de Rafael Faraco Benthien et de Frédéric Lebaron permettent de comprendre la réception du livre chez les « antiquisants » (p. 51), c'est-à-dire les latinistes et hellénistes français, et de manière plus sommaire chez les économistes. Rafael Faraco Benthien restitue dans un premier temps de manière brève l'histoire et la structure hétérogène des études gréco-latines. Cela lui permet d'étudier plus en détail les réceptions différenciées par ce groupe social du travail des durkheimiens. L'auteur conclut par l'analyse de la critique des Formes par deux de ces antiquisants, Salomon Reinach et Camille Jullian. Le texte de Frédéric Lebaron, s'il donne quelques informations brèves sur la réception des Formes par les économistes, vise plus explicitement à envisager sous quelles conditions les résultats généraux de l'ouvrage peuvent être transposés en enquêtes empiriques contrôlées à propos de l'espace économique contemporain. Selon lui, seules des enquêtes de ce type permettraient d'évaluer de manière précise la pertinence des hypothèses sur l'origine religieuse des concepts économiques et la séparation du domaine économique d'avec le reste de l'espace social (par la mobilisation de l'opposition entre sacré et profane). Ce texte stimulant permet ainsi d'effectuer une transition avec la deuxième partie du livre, consacrée aux lectures contemporaines des Formes. 
7 Les quatre contributions suivantes se centrent ainsi chacune sur un ou plusieurs thèmes plus ou moins explicitement développés dans l'ouvrage de Durkheim : CharlesHenry Cuin envisage le divin, Paul Dirkx le corps, William Watts Miller l'art, Nicolas Sembel les rapports entre religion, éducation, connaissance et sociologie. Ces lectures visent principalement, par le biais de contributions parfois théorico-empiriques, parfois plus nettement spéculatives, à stimuler le déroulement d'enquêtes empiriques. Ainsi, Charles-Henry Cuin mène une réflexion sur le statut du divin dans les Formes, qui se conclut par un paradoxe que l'on peut résumer de la manière suivante : si l'existence sociale de Dieu a été établie par l'auteur, peut-on dire qu'il cherchait par le biais de la sociologie à en prouver l'existence «réelle »? Au terme de leurs textes sur le corps et l'art, Paul Dirkx et William Watts Miller partagent une affirmation commune selon laquelle ces deux thèmes sont présents "partout et nulle part» (p.190) - ou " everywhere and nowhere» (p. 224) - dans les Formes. Enfin, Nicolas Sembel effectue une contribution importante à propos des rapports entre religion, éducation, connaissance et sociologie chez Durkheim. Souvent traités de manière séparée chez l'auteur, Nicolas Sembel s'attache d'abord à croiser ces différents thèmes, ce qui lui permet dans la suite de dégager une unité de la méthode de travail durkheimienne. Cette dernière est ensuite envisagée à travers l'exemple de la religion, puis détaillée en fonction des étapes qui la constituent: le tri, la hiérarchisation, la dynamisation, la généralisation.

8 Les quatre textes constituant la dernière partie analysent les sources utilisées dans l'élaboration des Formes. Le texte que livre Marcel Fournier s'interroge sur le poids de l'équipe formée autour de L'Année sociologique dans la production de Durkheim, et en particulier des Formes. À l'issue d'un texte assez court, l'auteur conclut à une influence de ses collaborateurs réunis autour de L'Année sociologique (notamment Marcel Mauss et Henri Hubert) sur Durkheim. Cette conclusion est à notre sens approfondie par le travail assez complémentaire de Matthieu Béra. La question que son article pose (celle du rôle de l'activité de recension de Durkheim au sein de L'Année sociologique dans la production des Formes), quoique différente, se traduit plus résolument par un dispositif d'enquête empirique élaboré, puisque l'auteur propose de croiser l'ensemble des comptes rendus publiés par Durkheim dans L'Année sociologique avec les références présentes dans les Formes. Sommairement, il conclut à une faible correspondance entre les deux activités, puisqu'une toute petite part des comptes rendus effectués sera finalement transformée en mention dans l'ouvrage. Enfin, Myron Achimastos et Frederico Delgado Rosa s'intéressent aux usages de la citation par Durkheim dans les Formes, à travers les cas de l'anthropologue britannique Carl Strehlow et du spécialiste des religions sémites de l'Antiquité William Robertson Smith. Ces contributions restituent de manière précise les enjeux historiques et sociaux permettant de comprendre les usages que Durkheim en fit.

9 Après avoir synthétisé les apports des différentes contributions constituant l'ouvrage, Nicolas Sembel conclut en faisant sienne l'injonction de Durkheim à « clore aussi l'ère des spécialités» (p.419), dans un but de poursuite de construction d'une sociologie résolument générale.

10 Cet ouvrage présente selon nous des qualités indéniables : sa dimension collective, sa construction précise, le soin apporté à son appareil critique, le matériau empirique mobilisé dans la plupart des contributions, ou encore l'effort de cumulativité des études de réception avec celles existant déjà, sont à notre sens à souligner. En revanche, on 
peut regretter que le prix élevé du livre et la composition exclusivement masculine de ses contributeurs fonctionneront sans doute comme des obstacles sociaux à son accès par le plus grand nombre. Malgré ces appréhensions, le volume nous semble en définitive constituer un outil précieux de compréhension précise et rigoureuse du travail central que constitue, toujours aujourd'hui, les Formes.

\section{AUTEURS}

MATTHIAS FRINGANT

Paris, EHESS, CESSP - CSE 